\title{
A TRIAD OF RULED SURFACES DEFINED BY RECIPROCAL POLARS*
}

\author{
BY A. F. CARPENTER
}

As an application of the theory of triads of ruled surfaces $\dagger$ in projective differential geometry, we shall consider here a system defined as follows. Let $l_{y z}$ be any line element of a general ruled surface $R_{y z}$, the points $P_{y}, P_{z}$ being the flecnodes of $l_{y z}$. The defining system of differential equations for $R_{y z}$ will have the form

$$
\begin{aligned}
& y^{\prime \prime}+p_{12} z^{\prime}+q_{11} y+q_{12} z=0 \\
& z^{\prime \prime}+p_{21} y^{\prime}+q_{21} y+q_{22} z=0
\end{aligned}
$$

where differentiation is with respect to a parameter $x$ and where $p_{12}{ }^{\prime}=2 q_{12}, p_{21}{ }^{\prime}=2 q_{21}$.

The planes osculating the flecnode curve $C$ of $R_{y z}$ at $P_{y}, P_{z}$ intersect in a line $l_{\psi \phi}$, the points $P_{\psi}, P_{\phi}$ of which are those in which $l_{\psi \phi}$ is cut by the tangents to $C$ at the respective points $P_{z}$, $P_{y}$. The expressions for $\psi, \phi$ are

$$
\psi=p_{12} z^{\prime}+q_{12} z, \quad \phi=p_{21} y^{\prime}+q_{21} y .
$$

The polar reciprocal of $l_{\psi \phi}$ with respect to the linear complex which osculates $R_{y z}$ along $l_{y z}$ is the line $l_{\eta \theta}$, the points $P_{\eta}, P_{\theta}$ of which are given by the expressions

(3) $\eta=p_{12} z^{\prime}+p_{12} p_{21} y+q_{12} z, \quad \theta=p_{21} y^{\prime}+q_{21} y+p_{12} p_{21} z$.

The points $P_{y}, P_{\psi}, P_{\eta}$ are collinear, as are also the points $P_{z}, P_{\phi}, P_{\theta}$.

A set of three lines $l_{y z}, l_{\psi \phi}, l_{\eta \theta}$, as thus defined, corresponds to each value of the parameter $x$ and determines in this way three ruled surfaces $R_{y z}, R_{\psi \phi}, R_{\eta \theta}$. From (1), (2) and (3) we obtain the defining system of differential equations for this triad of ruled surfaces. $¥$ It is

* Presented to the Society, June 13, 1931.

$\dagger$ A. F. Carpenter, Triads of ruled surfaces, Transactions of this Society, vol. 29 (1927), pp. 254-275. Hereafter denoted by the symbol T.

$\ddagger$ T, p. 256. 


$$
\begin{array}{ll}
y^{\prime}=-r_{21} y+\pi_{21} \phi, & \psi^{\prime}=3 r_{12} \psi+\left(\alpha-p_{12}\right) \phi-\alpha \theta, \\
z^{\prime}=-r_{12} z+\pi_{12} \psi, & \phi^{\prime}=\left(\beta-p_{21}\right) \psi+3 r_{21} \phi-\beta \eta, \\
y=\pi_{12} \pi_{21}(\eta-\psi), & \eta^{\prime}=p y-p_{12} p_{21} \alpha z+3 r_{12} \eta, \\
z=\pi_{12} \pi_{21}(\theta-\phi), & \theta^{\prime}=-p_{12} p_{21} \beta y-p z+3 r_{21} \theta,
\end{array}
$$

where*

$$
\begin{aligned}
p_{12}^{2} p_{21} \alpha & =p_{12}^{2} q_{22}-p_{12} q_{12}^{\prime}+3 q_{12}^{2}, \\
p_{12} p_{21}^{2} \beta & =p_{21}^{2} q_{11}-p_{21} q_{21}^{\prime}+3 q_{21}^{2}, \\
p & =p_{12} q_{21}-p_{21} q_{12}, \quad \pi_{12} p_{12}=\pi_{21} p_{21}=1, \\
p_{12} r_{12} & =q_{12}, \quad p_{21} r_{21}=q_{21} .
\end{aligned}
$$

The three lines $l_{y z}, l_{\psi \phi}, l_{\eta \theta}$ determine a quadric $Q$. On each of these lines there will be two points such that tangents at these points to the respective surfaces $R_{y z}, R_{\psi \phi}, R_{\eta \theta}$ will be lines of that regulus of $Q$ whose lines intersect $l_{y z}, l_{\psi \phi}, l_{\eta \theta}$. These points are found by factoring the respective quadratic covariants $\dagger$

(6) $p_{21} y^{2}-p_{12} z^{2}, \quad \beta \psi^{2}-\alpha \phi^{2}, \quad \beta \eta^{2}+2 p \pi_{12} \pi_{21} \eta \theta-\alpha \theta^{2}$.

In this connection we remark that the first of these covariants determines the complex points of $l_{y z}$.

On each of the lines $l_{y z}, l_{\psi \phi}, l_{\eta \theta}$ lie two points at which intersector tangents§ to the surfaces $R_{y z}, R_{\psi \phi}, R_{\eta \theta}$ will also be tangents to the respective surfaces $R_{\psi \phi}, R_{\eta \theta}, R_{y z}$. These points are given by the respective covariants $\Phi$

$$
\begin{gathered}
\left(p_{21} \alpha-p_{12} \beta\right) y z, p\left(\beta \psi^{2}-\alpha \phi^{2}\right), \\
p \pi_{12} \eta^{2}+\left(p_{21} \alpha-p_{12} \beta\right) \eta \theta-p \pi_{21} \theta^{2} .
\end{gathered}
$$

If the invariant $p_{21} \alpha-p_{12} \beta \neq 0$, then from the first expression in (7) it follows that the intersector tangents to $R_{y z}$ at the flecnodes of

* We shall assume that $p_{12}$ and $p_{21}$ do not vanish identically, that is, that neither branch of the flecnode curve of $R_{y z}$ is a straight line.

$\dagger$ T, p. 269 et seq.

¥ A. F. Carpenter, Ruled surfaces whose flecnode curves have plane branches, Transactions of this Society, vol. 16 (1915).

$\S \mathrm{E}$. P. Lane, Ruled surfaces with generators in one-to-one correspondence, Transactions of this Society, vol. 25 (1923).

ๆ T, p. 269 et seq. 
$l_{y z}$ are tangent to $R_{\psi \phi}$. If $p_{21} \alpha-p_{12} \beta \equiv 0$, then the intersector tangents to $R_{y z}$ at all points of $l_{y z}$ are tangent to $R_{\psi \phi}$. This conclusion can be verified in the following way.

Let $u=y+\lambda z$ be the expression for the general point on $l_{y z}$ and let us choose for system of reference the tetrahedron $P_{y} P_{z} P_{\rho} P_{\sigma}$, where the unit point is so chosen that all coordinates of the four vertices are zero except $y_{1}=z_{2}=\rho_{3}=\sigma_{4}=1$, and where

$$
\rho=2 y^{\prime}+p_{12} z, \sigma=2 z^{\prime}+p_{21} y .
$$

Then with the help of equations (4) and (8) the equation of the tangent plane to $R_{y z}$ at $P_{u}$ is found to be

$$
\lambda x_{3}-x_{4}=0 .
$$

This plane cuts $l_{\psi \phi}$ in the point $P_{v}$, where

$$
v=\psi+k \phi, p_{21} k \lambda=p_{12} .
$$

The equation of the tangent plane to $R_{\psi \phi}$ at $P_{v}$ is, similarly,

$p_{21} \alpha \lambda x_{1}-p_{12} \beta x_{2}-\left(p_{12}^{2} \beta+2 q_{21} \alpha \lambda\right) x_{3}+\left(p_{21}^{2} \alpha \lambda+2 q_{12} \beta\right) x_{4}=0$, and the point $P_{u}$ lies on this plane for all values of $\lambda$, if, and only if, $p_{21} \alpha-p_{12} \beta \equiv 0$.

In a previous paper by the author* it was proved that the osculating planes of the flecnode curve, the complex curve and the harmonic curve of $R_{y z}$ at the points in which these curves cut any line element $l_{y z}$, will form an axial pencil if, and only if, $p_{21} \alpha-p_{12} \beta=0$. In view of what precedes we may state the following theorem.

Theorem 1. If the planes osculating the flecnode curve, the complex curve, and the harmonic curve of a general ruled surface $R$ at the points of intersection of these curves with each line element of $R$ form an axial pencil, then $R$ and the ruled surface $S$ generated by the axis of this pencil of planes are the focal surfaces of the congruence of intersector tangents of $R($ or $S)$ with respect to $S($ or $R$ ).

If the invariant $p \neq \equiv 0$, then from (6) and (7) it results that the two points of $l_{\psi \phi}$ at which lines of $Q$ are tangent to $R_{\psi \phi}$ are also the points at which intersector tangents of $R_{\psi \phi}$ are tangent to $R_{\eta \theta}$. If $p \equiv 0$, then the intersector tangents to $R_{\psi \phi}$ at all points of $l_{\psi \phi}$ are

* A. F. Carpenter, $A$ theorem on ruled surfaces, this Bulletin, vol. 34 (1928), pp. $479-481$. 
tangent to $R_{\eta \theta}$. This can be verified as above. But if $p \equiv 0$, then $R_{y z}$ is identically self-dual.* We can sum up these results in the following statement.

THEOREM 2. Let the planes osculating the flecnode curve of a general ruled surface $R$ at the two points of intersection of this curve with a line element $l$ of $R$ intersect in $s$, and let $t$ be the polar reciprocal of $s$ with respect to the linear complex osculating $R$ along l. Then if $R$ is identically self-dual, the two ruled surfaces $S$ and $T$ generated by $s$ and $t$ will be the focal surfaces of the congruence of intersector tangents of $S$ (or $T$ ) with respect to $T$ (or $S$ ).

If both $p_{21} \alpha-p_{12} \beta \equiv 0$ and $p \equiv 0$, then the third covariant of (7) vanishes identically. Under these conditions, $R_{y z}$ is a quadric. $\dagger$

Two of the intersector tangents of $R_{y z}$ at points of $l_{y z}$ must be asymptotic tangents of $R_{y z}$. We find these points as follows. In order that the curve $C_{u}$ generated by $P_{u}$ be an asymptotic curve of $R_{y z}, \lambda$ must satisfy the differential equation

$$
2 \lambda^{\prime}+p_{21} \lambda^{2}-p_{12}=0 \text {. }
$$

By the use of (4) and (10), we find

$$
u^{\prime}=-r_{21} y-\frac{1}{2}\left(p_{21} \lambda^{2}+2 r_{12} \lambda-p_{12}\right) z+\pi_{12} \lambda \psi+\pi_{21} \phi .
$$

Now the asymptotic tangent at $P_{u}$ will cut $l_{\psi \phi}$ if $u^{\prime}+\mu u$ is linear in $\psi$ and $\phi$. It follows that

$$
u-r_{21}=0, p_{21} \lambda^{2}-2 \mu \lambda+2 r_{12} \lambda-p_{12}=0,
$$

and therefore

$$
p_{21} \lambda^{2}-2\left(r_{21}-r_{12}\right) \lambda-p_{12}=0 .
$$

The values of $\lambda$ from (12) determine the two values of $u$ sought. The corresponding quadratic covariant is found to be

$$
p_{21} y^{2}+2\left(r_{21}-r_{12}\right) y z-p_{12} z^{2}=\pi_{21} P Q,
$$

where

$$
\begin{gathered}
P=p_{21} y+U z, Q=p_{21} y+V z, U=r_{21}-r_{12}+r, V=r_{21}-r_{12}-r, \\
r=\left[\left(r_{21}-r_{12}\right)^{2}+p_{12} p_{21}\right]^{1 / 2} .
\end{gathered}
$$

* E. J. Wilczynski, Projective Differential Geometry, p. $144 ; p$ is a factor of the invariant.

$\dagger$ Wilczynski, loc. cit., p. 150. 
The two points in which these asymptotic tangen ts cut $l_{\psi \phi}$ are given by $\pi_{12} \lambda_{1} \psi+\pi_{21} \phi, \pi_{12} \lambda_{2} \psi+\pi_{21} \phi$, where $\lambda_{1}, \lambda_{2}$ are the roots of (12), and the corresponding covariant is found to be

$$
p_{21} \psi^{2}-2\left(r_{21}-r_{12}\right) \psi \phi-p_{12} \phi^{2}=\pi_{12} C D,
$$

where $C=p_{21} \psi-V \phi, D=p_{21} \psi-U \phi$.

By proceeding as above we find, for the points of $l_{y z}$ at which asymptotic tangents to $R_{y z}$ cut $l_{\eta \theta}$, the covariant

$$
p_{21} y^{2}-2\left(r_{21}-r_{12}\right) y z-p_{12} z^{2}=\pi_{21} R S,
$$

where $R=p_{21} y-U z, S=p_{21} y-V z$, and for the points in which these tangents cut $l_{\eta \theta}$ the covariant

$$
p_{21} \eta^{2}+2\left(r_{21}-r_{12}\right) \eta \theta-p_{12} \theta^{2}=\pi_{21} A B,
$$

where $A=p_{21} \eta+V \theta, B=p_{21} \eta+U \theta$.

By comparison of (13), (14), (15), (16) it results that the line $l_{y z}$ is cut by those faces of the tetrahedron $A B C D$ opposite the vertices $A, B, C, D$ in the respective points $P, Q, R, S$. It is also seen that $P_{y}, P_{z}$ separate harmonically both pairs of points $P, R$ and $Q, S$.

Since $r_{21}-r_{12} \equiv 0$ implies $p \equiv 0$, and conversely, it results that if $R_{y z}$ is identically self-dual the asymptotic tangents to $R_{y z}$ at the complex points of $l_{y z}$ intersect both $R_{\psi \phi}$ and $R_{\eta \theta}$ and are thus rulings of the quadric determined by $l_{y z}, l_{\psi \phi}, l_{\eta \theta}$.

There exist, in general, for the three lines $l_{y z}, l_{\psi \phi}, l_{\eta \theta}$, two closed intersector sequences of order one.* The pairs of points in which these lines are cut by these sequences are given by the respective covariants

$$
\begin{aligned}
& p_{21} \alpha \beta y^{2}+p\left(\pi_{12} \alpha+\pi_{21} \beta\right) y z-p_{12} \alpha \beta z^{2}, \\
& p_{21} \alpha \beta \psi^{2}-p\left(\pi_{12} \alpha+\pi_{21} \beta\right) \psi \phi-p_{12} \alpha \beta \phi^{2}, \\
& p_{12} \beta^{2} \eta^{2}+p\left(\pi_{12} \alpha+\pi_{21} \beta\right) \eta \theta-p_{21} \alpha^{2} \theta^{2} .
\end{aligned}
$$

The existence of a closed intersector sequence of order two is assured by the vanishing of the invariant

$$
p \pi_{12} \pi_{21}\left(p_{21} \alpha-p_{12} \beta\right),
$$

and this closure property holds for all such sequences. From (18) it follows that, under the conditions of either Theorem 1 or Theorem 2, all intersector sequences of order two are closed.

*T, pp. 272, 273. 
All intersector sequences of order three will be closed if the invariant

$$
p^{2}\left(p_{21}^{2} \alpha^{2}+p_{12}^{2} \beta^{2}\right)+2 p_{12}^{3} p_{21}^{3} \alpha^{2} \beta^{2}
$$

vanishes. This can happen either if $p=0, \alpha=0$ (or $\beta=0$ ), or $\alpha=0, \beta=0$. In the first case one branch of the flecnode curve of $R_{y z}$ is plane, $(\alpha=0)$, and $R_{\psi \phi}$ degenerates into the tangents of a plane curve. In the second case both branches of the flecnode curve of $R_{y z}$ are plane and $R_{\psi \phi}$ degenerates into a straight line.

It is obvious that in the preceding developments the order of the lines $l_{y z}, l_{\psi \phi}, l_{\eta \theta}$ can be reversed without in any way affecting results. The analysis would be based upon a system of firstorder equations of the same type as (4), (5) and obtainable from (4), (5) by simple processes.

The University of Washington

\section{NOTE ON THE REDUCIBILITY OF ALGEBRAS WITHOUT A FINITE BASE*}

BY M. H. INGRAHAM

It is the purpose of this note to discuss the reducibility of linear associative algebras which are not assumed to possess a finite base. J. H. M. Wedderburn, $\uparrow$ in seeking to generalize certain theorems on the structure of an algebra, has considered algebras in which restrictions are placed upon the character of the idempotent elements. The summations involved in his study need not be finite. This seems to be one natural line of attack.

J. W. Young $\ddagger$ has approached the subject from the point of view of the groups involved. His definition of a finite algebra is, however, unsatisfactory, not being sufficiently restrictive.

I have studied infinite algebras in connection with the results that can be obtained by a use of the "axiom of choice" and the theory of transfinite ordinals. This note, however, does not

* Presented to the Society, December 31, 1928.

$\dagger$ J. H. M. Wedderburn, Algebras which do not possess a finite base, Transactions of this Society, vol. 26 (1924), pp. 395-426.

$¥ \mathrm{~J}$. W. Young, $A$ new formulation for general algebras, Annals of Mathematics, vol. 29 (1927), pp. 47-60. See particularly p. 60 . 